\title{
Soluble Liver Antigen/Liver and Pancreas, an Antigen in Autoimmune Hepatitis Patients: Influence on Selenocysteine Synthesis and its Complex with HSP70
}

\author{
Yuta Tanaka, ${ }^{a}$ Takashi Osaka, ${ }^{a}$ Asuka Hattori, ${ }^{a}$ Yasuko Murakami, ${ }^{b}$ Tamiko Matsufuji, ${ }^{b}$ \\ Senya Matsufuji, ${ }^{b}$ Takashi Shinkawa, ${ }^{c}$ Toshiaki Isobe, ${ }^{c}$ and Takaharu Mizutani ${ }^{*}, a$ \\ ${ }^{a}$ Graduate School of Pharmaceutical Sciences, Nagoya City University, 3-1 Tanabe-dori, Mizuho-ku, Nagoya 467-8603, Japan, ${ }^{b}$ Jikei \\ University School of Medicine, Nishishinbashi, Minato-ku, Tokyo 105-8461, Japan, and ${ }^{c}$ Faculty of Sciences, Tokyo Metropolitan \\ University, Minamiosawa, Hachiouji, Tokyo 192-0397, Japan
}

(Received June 5, 2007; Accepted September 11, 2007; Published online October 1, 2007)

\begin{abstract}
Autoimmune hepatitis (AIH) serum contains autoantibodies against soluble liver antigen/liver and pancreas (SLA/LP), a protein that interacts with the selenocysteine $(\mathrm{Sec})-\mathrm{tRNA}^{\mathrm{Sec}}$. We investigated the influence of AIH serum on Sec production. Two (\#3 and \#4) of four AIH sera inhibited its synthesis, with serum \#4 showing the strongest inhibition. This did not parallel the level of antibodies to SLA/LP (anti-SLA/LP) in sera, because the titer in serum \#3 was higher than that in serum \#4. Thus, we found that AIH inhibited Sec synthesis but we could not conclude that this inhibition depended on an autoantibody against SLA/LP. To clarify the function of SLA/LP, we prepared an antibody against an SLA/LP peptide. Inhibition of Sec synthesis by this anti-peptide antibody was incomplete and sometimes did not occur. Absorption of autoantibodies in the AIH serum with the SLA/LP protein did not have any effects on Sec synthesis by Sec synthase (SecS) which converts Seryl (Ser)-tRNA ${ }^{\text {Sec }}$ to Sec-tRNA ${ }^{\text {Sec }}$. We then used SLA/LP instead of active bovine SecS but we could not detect SecS activity in SLA/LP itself. Next, we studied the distribution of SLA/LP in HEK293 cells transfected with an SLA/LP expression vector. Immunofluorescence microscopy detected SLA/LP expression in the cytoplasm, but not the nucleus of HEK293 cells. Higher levels of SLA/LP in the cytosol did not correlate with higher Sec production, which remained identical to mock-transfected cells. Interestingly, we found that SLA/LP formed a complex in the cytosol of HEK293 cells. This complex was purified and the components analyzed using Nano-LC and MS/MS, which we identified as HSP70 and tubulin. Altogether, our data suggest that SLA/LP may play an indirect role to produce Sec in the selenoprotein synthesis machinery.
\end{abstract}

Key words — SLA/LP, HSP70, autoimmune hepatitis, tRNA, selenocysteine, Selenium

\section{INTRODUCTION}

Autoimmune hepatitis (AIH) is a disease of unknown etiology characterized by hypergammaglobulinemia, liver-related autoantibodies, and an association with a favorable response to immunosuppression. The current classification of $\mathrm{AIH}$ and the several autoantibodies/target autoantibodies found in this disease are reported. AIH is subdivided into two major types: AIH type 1 and AIH type 2. ${ }^{1)}$ AIH type 1 is characterized by the detection of smooth muscle autoantibodies (SMA) and/or antin-

*To whom correspondence should be addressed: Graduate School of Pharmaceutical Sciences, Nagoya City University, 3-1 Tanabe-dori, Mizuho-ku, Nagoya 467-8603, Japan. Tel. \& Fax: +81-52-836-3490; E-mail: mizutani@phar.nagoyacu.ac.jp uclear antibodies (ANA). Antineutrophil cytoplasmic autoantibodies (ANCA), antibodies against the asialoglycoprotein receptor (anti-ASGP-R) and antibodies to soluble liver antigens/liver and pancreas (anti-SLA/LP) may be useful for the identification of individuals who are seronegative for ANA/SMA. AIH type 2 is characterized by the presence of specific autoantibodies against liver and kidney misrosomal antigens (anti-LKM) and/or autoantibodies against liver cytosol 1 antigen.

In 1992, Gelpi et al. ${ }^{2)}$ found an autoantibody against a selenocysteine (Sec)-tRNA-protein complex in AIH patients and later the sequence of the protein in the complex was reported. ${ }^{3)}$ This protein was postulated to be the translation elongation factor specific to Sec-tRNA ${ }^{\mathrm{Sec}}$. ${ }^{4)}$ Two research groups ${ }^{5,6)}$ reported an SLA/LP amino acid 
sequence identical to the protein described by Gelpi et al. $^{2)}$ From these results, it is clear that SLA/LP in AIH type 1 patients is the protein binding to tRNA $^{\text {Sec }}$; however, the function of SLA/LP in Sec synthesis is not clear. From a comparison of the SLA/LP amino acid sequence of protein structures in the protein database, a bioinformatic approach suggested that SLA/LP might be an enzyme containing pyridoxal phosphate as a cofactor, similar to serine hydroxymethyltransferase. ${ }^{7)}$ Thus it was speculated that SLA/LP is a human Sec synthase (SecS), although there was no evidence to support this. The concept of the existence of autoantibodies against factors of the translation machinery was not peculiar considering that many autoantibodies against aminacyl-tRNA synthetases and even tRNAs have been reported. ${ }^{8)}$

Selenium is an essential trace element and deficiency causes an endemic disease, Keshan disease. 9) Meanwhile, $\mathrm{Se}$ is known to be toxic in livestock, in which it causes loco disease. Se is found in selenoproteins as $\mathrm{Sec}$, which is recognized as the 21st amino acid. It is encoded by a reprogrammed UGA codon recognized by the specific tRNA ${ }^{\text {Sec }} .^{10,11)}$ Sec-tRNA and/or the relevant translation apparatus are found in eukarya, bacteria, and archaea, but not in higher plants and yeast. ${ }^{12)}$ Selenocysteine biosynthesis starts from serine, as demonstrated in the case of GPx in which the carbon backbone of Sec originates from ${ }^{14} \mathrm{C}$-Ser. Subsequently, $\left[{ }^{75} \mathrm{Se}\right] \mathrm{Sec}$ can be prepared in vitro using the natural tRNA ${ }^{\mathrm{Ser}}$ or the corresponding T7 transcript, bovine liver cytosol as the enzyme source, and $\left[{ }^{75} \mathrm{Se}\right] \mathrm{H}_{2} \mathrm{Se}$ as the Se donor. ${ }^{13)}$ In bacteria, the SecS that converts the Ser-tRNA ${ }^{\mathrm{Sec}}$ to Sec-tRNA ${ }^{\mathrm{Sec}}$ has been characterized. ${ }^{14)}$ In the eukaryotic tRNA ${ }^{\mathrm{Sec}}$, the identity elements necessary for selenylation in vitro are the 9-bp long aminoacyl stem and the 6-bp long. ${ }^{15-17)}$ The biologically active selenium donor is selenophosphate that is produced by the enzyme selenophosphate synthetase (SPS) from $\mathrm{H}_{2} \mathrm{Se}$ and ATP. ${ }^{18)}$ Sec-tRNA ${ }^{\text {Sec }}$ is then brought to the A site of ribosomes by the specialized translation elongation factor called $\mathrm{EF}_{\mathrm{Sec}}$ in mammals. ${ }^{19,20)}$ In eukarya, discrimination of the UGA Sec from the UGA stop codon is accomplished by a stem-loop structure in the $3^{\prime}$-untrasnlated region of selenoprotein mRNAs called SECIS, recognized by the SECIS-specific binding protein SBP2. This protein interacts with the $\mathrm{EF}_{\mathrm{Sec}}-\mathrm{Sec}-\mathrm{tRNA}{ }^{\mathrm{Sec}}{ }^{21)}$

We report here the inhibition of Sec synthesis by sera from AIH patients. We also show the influ- ence on Sec production of a serum directed against a $C$-terminal peptide of SLA/LP. Study of the distribution of SLA/LP in HEK293 cells established that it localizes in the cytosol.

\section{MATERIALS AND METHODS}

Sera from AIH Patients — The four female AIH type 1 sera used were \#1, \#2, \#3, and \#4 from patients 57, 50, 63, and 48 years old, respectively. AIH type 1 was confirmed by an ANA-positive reaction. \#1 has Sjögren syndrome and \#2 Hashimoto disease; these two patients had been treated with steroids. Sera from \#3 and \#4 also contained antitRNA antibodies. All four patients were negative for hepatitis virus B and C. Permission to use the sera was given by each patient. We also obtained sera from healthy volunteers as a control, supplied from the Aichi Prefectural Blood Center of the Japanese Red Cross.

ELISA for Estimation of Anti-SLA/LP — The SLA/LP amino acid sequence used for aiming the anti-peptide antibody was RKERSKESDDNYDKTEDVD (positions 402-420). ${ }^{22)}$ This peptide was conjugated with keyhole limpet hemocyanin and then injected four times into two rabbits. Enzymelinked immunosorbent assay (ELISA) for the estimation of the antibody level was performed by the standard method, ${ }^{23}$ ) as follows: The peptide was adsorbed onto 96-well plates, and then AIH or rabbit serum was used as the primary antibody source to measure the level of antibody in the serum. Secondary antibodies (goat) conjugated with horse radish peroxidase (HRP) for human and rabbit globulins were also used.

Inhibition of Sec Synthesis —_ Sec was produced in vitro as follows: ${ }^{13)}$ Assay of $\left[{ }^{75} \mathrm{Se}\right] \mathrm{Sec}$ production was carried out in a reaction mixture $(50 \mu \mathrm{l})$ containing T7 transcript tRNA ${ }^{\mathrm{Sec}}(0.2 \mu \mathrm{g})$, ATP $5 \mathrm{mM}$, Ser $0.4 \mathrm{mM},\left[{ }^{75} \mathrm{Se}\right] \mathrm{H}_{2} \mathrm{Se} 0.1 \mu \mathrm{M}(0.02 \mu \mathrm{Ci})$, seryl-tRNA synthetase (SerRS), and Sec-synthesizing enzymes (partially purified cytosol enzymes from bovine liver), according to a previous report. ${ }^{13)}$ After incubation for $2 \mathrm{hr}$ at $30^{\circ} \mathrm{C}$, $\left[{ }^{75} \mathrm{Se}\right] \mathrm{Sec}-\mathrm{tRNA}$ was collected by ethanol precipitation, and the precipitate was treated for $30 \mathrm{~min}$ with $5 \mu \mathrm{l}$ of mild alkaline solution. For inhibition experiments, Sec-synthesizing enzymes were preincubated with serum for $30 \mathrm{~min}$ at $0^{\circ} \mathrm{C}$ before the assay of $\left[{ }^{75} \mathrm{Se}\right] \mathrm{Sec}$ production. The amino acid released from aminoacyltRNA $^{\text {Sec }}$ was spotted onto a silica gel G TLC 
plate. Authentic Sec was also spotted and cochromatographed. Amino acids were chromatographed in $n$-butanol : acetic acid: water $(4: 1: 1)$. The position of Sec was revealed by staining with ninhydrin. $\left[{ }^{75} \mathrm{Se}\right] \mathrm{Sec}$ was detected on TLC plates with a Fuji (Tokyo, Japan) BAS 2500 image analyzer. Secsynthesizing enzymes from bovine liver and sometimes from human liver cytosol of products supplied by Gentest (Woburn, MA, U.S.A.) were used.

Construction of the SLA/LP Expression Vector-Human SLA/LP was expressed in Escherichia coli (E. coli) from plasmid pET-14bSLA/LP ${ }^{4}$ or expression in HEK293 cells, the SLA/LP insert from the previous plasmid was introduced into pCAGGS-3FLAG-6His-SLA/LP having FLAG and 6His tags. HEK293 cells were cultured at $5 \times 10^{5}$ cells in a 9-cm dish for $16 \mathrm{hr}$ and then transfected with pCAGGS-3FLAG-6HisSLA/LP. After $48 \mathrm{hr}$, cells were collected and lysed in a buffer containing Tris- $\mathrm{HCl} 50 \mathrm{mM}$ at $\mathrm{pH} 7.5$, $\mathrm{NaCl} 150 \mathrm{mM}, 1 \% \mathrm{NP}$, and complete mini EDTAfree (Roche, Mannheim, Germany) for $30 \mathrm{~min}$ on ice. Cytosol and nucleus fractions were prepared from this lysate according to the standard methods. Western Blotting — Western blotting was carried out according to a previous report. ${ }^{24)} \mathrm{SecS}$ highly purified from liver and a SLA/LP complex in HEK293 cells was analyzed by standard SDSPAGE and blotted onto a polyvinylidene difluoride (PVDF) membrane. Proteins on the membrane were immunostained by serum from patient \#4, anti-SLA/LP peptide, or anti-FLAG. After staining with an HRP-conjugated goat secondary antibody (MBL, Nagoya, Japan), bands were detected by ECL kit (Amersham Bioscience, Buckinghamshire, England) and analyzed with a Lumino-image Analyzer LAS-1000 (Fuji).

Immunofluorescence Microscopy — HEK293 cells were cultured on a slide glass and the expression vector was transfected to the glass. Cells were washed with phosphate-buffered saline (PBS) and fixed with $4 \%$ paraformaldehyde for $15 \mathrm{~min}$ at room temperature. The glass was washed with $0.2 \%$ Triton X-100 in PBS and then blocked with $2 \%$ bovine serum albumin (BSA) in PBS for $1 \mathrm{hr}$. The primary antibody against FLAG was added and incubated for $1 \mathrm{hr}$. The sample was washed three times with PBS and reacted with the secondary antibody conjugated with fluorescein isochiocyanate (FITC) for $1 \mathrm{hr}$ at room temperature. It was then washed five times with PBS and stained with propidium iodide in $50 \%$ glycerol for $3 \mathrm{~min}$.
The pattern of fluorescence in cells was measured with immunofluorescence microscopy (LSM 510, Fujix).

Purification and Analysis of an SLA/LP Complex — Cytosol (5 mg protein) from HEK293 cells expressing the FLAG- and 6His-tagged SLA/LP was mixed with mouse IgG-Agarose $(50 \mu \mathrm{l})$, to give $1 \mathrm{ml}$ with PBS and mixed for $1 \mathrm{hr}$ at $4^{\circ} \mathrm{C}$. A $5000 \mathrm{~g}$ supernatant of the above solution was mixed with $50 \mu \mathrm{l}$ of anti-FLAG M2 affinity beads (SIGMA, St, Louis, MO, USA) for $1 \mathrm{hr}$ at $4^{\circ} \mathrm{C}$. The beads were collected by centrifugation and washed with a buffer containing Tris- $\mathrm{HCl} 50 \mathrm{mM}$ at $\mathrm{pH} 7.5, \mathrm{NaCl} 160 \mathrm{mM}, 5 \%$ glycerol, and $0.05 \%$ Triton X-100. The protein bound on the beads was eluted with eluting solution A made of $0.25 \mathrm{mg} / \mathrm{ml}$ $3 \times$ FLAG peptide, Tris- $\mathrm{HCl} 50 \mathrm{mM}, \mathrm{pH} 7.5, \mathrm{NaCl}$ $160 \mathrm{mM}, 5 \%$ glycerol, $0.05 \%$ Triton X-100. The eluate was further purified with Ni beads (Clontech, Takara-bio, Tokyo, Japan). Purified SLA/LP complex was stored at $-80^{\circ} \mathrm{C}$.

The SLA/LP complex was analyzed with $10 \%$ polyacrylamide gel electrophoresis (PAGE). To detect a protein pattern, the gel was silver stained. ${ }^{25}$ ) Meanwhile, for protein analysis of protein in the SLA/LP complex, proteins were modified to RCM and treated with methanol-chloroform. The precipitate was digested with trypsin and analyzed with nano-liquid chromatography (LC)-MS/MS. Peaks were analyzed with Mascott Ref Seq-human and calculated with STEM, according to the previous method. ${ }^{26)}$

\section{RESULTS}

\section{AIH Sera Inhibit Selenocysteine Synthesis}

Earlier work by others reported that SLA/LP was isolated as a tRNA ${ }^{\mathrm{Sec}}$-bound factor and was considered as the translation elongation factor specific to Sec-tRNA ${ }^{\text {Sec }}$. ${ }^{\text {) }}$ The SLA/LP amino acid sequence contains blocks of homology to cofactors of the pyridoxal phosphate family. ${ }^{7)}$ Therefore it is possible that SLA/LP is the enzyme converting the Ser-tRNA $^{\text {Sec }}$ to Sec-tRNA ${ }^{\mathrm{Sec}}$, becausee SecS in $E$. coli contains a bound pyridoxal phosphate. We have shown earlier SecS activity in mammals. ${ }^{13}$ Here, we asked whether AIH sera could inhibit Sec synthesis. The results are shown in Fig. 1, including the TLC pattern showing the autoradiography of $\left[{ }^{75} \mathrm{Se}\right] \mathrm{Sec}$ spots. Figure 1 shows that sera \#1 and \#2 did not inhibit Sec production, but that sera \#3 and 


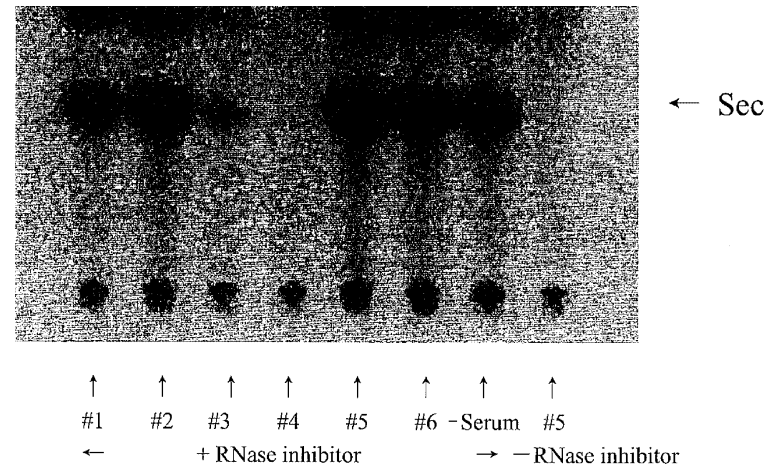

Fig. 1. TLC Pattern of $\left[{ }^{75} \mathrm{Se}\right] \mathrm{Sec}$

Inhibitory patterns of Sec sythesis by AIH patient sera \#1, \#2, $\# 3$, and \#4, and normal sera \#5 and \#6. The right lane lacked RNase inhibitor.

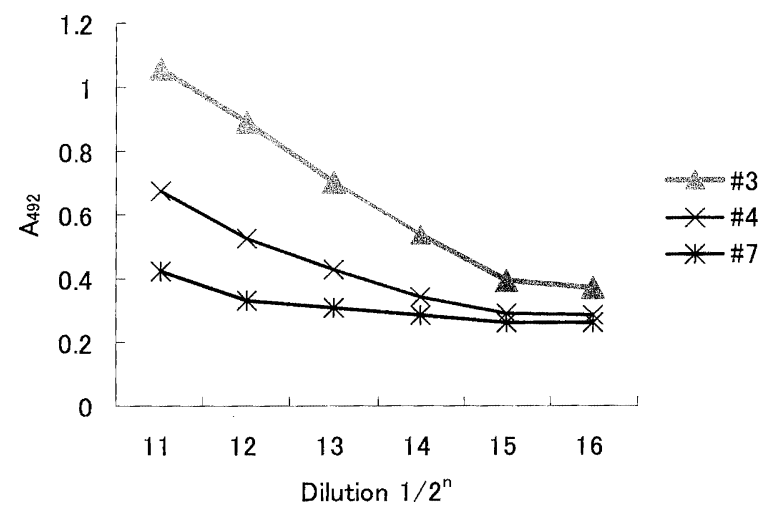

Fig. 2. Titration Patterns with SLA/LP for AIH Sera (\#3 and \#4) and Normal Serum (\#7)

\#4 clearly did. In this experiment, $1 \mu \mathrm{l}$ of serum was used for preincubation with enzymes and then the reaction was started by the addition of this mixture to the substrate solution. For serum \#3, a weak radioactivity signal was found in the $\left[{ }^{75} \mathrm{Se}\right] \mathrm{Sec}$ spot on TLC showing mild inhibition. Meanwhile, serum \#4 completely inhibited Sec formation. We next measured the antibody level against SLA/LP in sera \#3 and \#4 using ELISA. As shown in Fig. 2, it was higher in serum \#3 than in serum \#4. The experiment with the protein-A column provided evidence that inhibition by serum \#4 was caused by $\operatorname{IgG}$ (data not shown). We also confirmed that the inhibition by AIH sera was not caused by the serum ribonuclease that could hydrolyze the $\mathrm{RNNA}^{\mathrm{Sec}}$ in the reaction mixture for Sec production, as the level of ribonuclease in AIH sera was the same that in the four AIH sera and in the control sera. We concluded that AIH sera can inhibit Sec production, but it was not clear whether the inhibition depended on SLA/LP.

\section{Study with SLA/LP and SLA/LP Anti-peptide Antibody}

To confirm that SLA/LP is a component of the SecS system, we prepared an SLA/LP anti-peptide antibody against the $C$-terminal position of SLA/LP. The globulin fraction was obtained by fractional precipitation with ammonium sulfate. Figure $3 \mathrm{~A}$ shows the results of the inhibition of Sec formation by anti-peptide antibody, as well as normal globulin. Sec formation was not inhibited at globulin $10 \mu \mathrm{g}$ and was inhibited by $50 \%$ at globulin $30 \mu \mathrm{g}$ from anti-peptide and nonimmune sera. Formation was inhibited at globulin $300 \mu \mathrm{g}$ from both anti-peptide and nonimmune sera. These results were confirmed in repeated experiments. Thus SLA/LP anti-peptide globulin showed similar inhibition to that of nonimmune rabbit globulin. Next, SLA/LP in the active cytosolic fraction was absorbed through an SLA/LP anti-peptide globulin column. The results are shown in Fig. 3B. The activity of the fraction arising from anti-SLA/LP binding was $50 \%$ of that obtained by binding nonimmune globulin. We could also clearly detect Sec-producing activity after this absorption. From these results, we consider that SLA/LP plays a role in enhancing Sec formation in Sec-producing machinery, and that SLA/LP itself is not SecS.

We further studied the influence of SLA/LP on Sec synthesis activity. We asked whether binding of SLA/LP to antisera would inhibit SecS activity. Figure $3 \mathrm{C}$ shows that SLA/LP had no influence on SecS activity. Lanes 2, 3, and 4 are in the presence of sera $\# 3$, \#4, and \#7 in keeping with the results displayed in Fig. 1. This inhibiting activity of the serum from patient \#4 was not absorbed by SLA/LP protein and no SecS activity could be recovered with the addition of SLA/LP 30-60 $\mu \mathrm{g}$ in duplicate (lanes 5-8). Meanwhile, in lanes 9 and 10, SLA/LP did not influence $\mathrm{SecS}$ activity. SLA/LP itself $(30-100 \mu \mathrm{g}) \mathrm{did}$ not carry SecS activity (lanes 11-13). Thus SLA/LP did not interact with SecS activity or the inhibitory activity of the antiserum. Figure 3D confirms that SLA/LP $0.6 \mu \mathrm{g}$ did not absorb the inhibitory activity of the antibody in the AIH serum. The upper panel is without SLA/LP and the lower is with SLA/LP protein. The inhibitory effect of patient \#4 AIH serum was not affected by the addition of SLA/LP. Thus we could not detect any Sec-producing activity in SLA/LP. The SLA/LP anti-peptide antibody also did not lead to complete inhibition of Sec synthesis.

\section{Distribution of SLA/LP in HEK293 Cells}

We next studied the distribution of native 
(A)

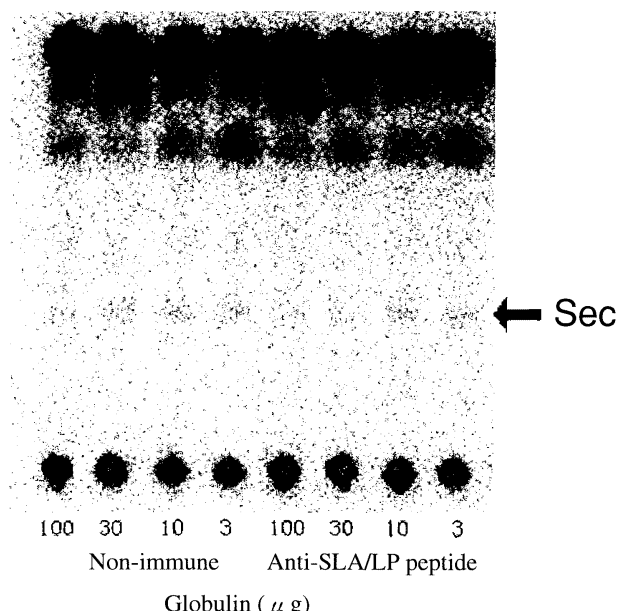

(B)

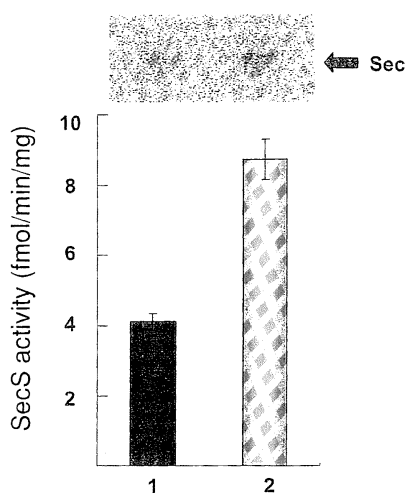

(C)

\begin{tabular}{|c|c|c|c|c|c|c|c|c|c|c|c|c|c|c|}
\hline Lane & 1 & 2 & 3 & 4 & 5 & 6 & 7 & 8 & 9 & 10 & 11 & 12 & 13 & 14 \\
\hline Sec & ma & & & & & & & & . & & & & & \\
\hline $\operatorname{SLA} / L P(\mu g)$ & - & - & - & - & 60 & 30 & 60 & 30 & 60 & 30 & 60 & 30 & 100 & - \\
\hline SecS & + & + & + & + & + & + & + & + & + & + & - & 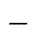 & - & - \\
\hline RNase inhibitor & - & + & + & + & + & + & + & + & - & - & - & 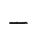 & . & - \\
\hline Serum $(1 \mu l)$ & - & \#3 & $\# 4$ & $\# 7$ & \#4 & $\# 4$ & $\# 4$ & $\# 4$ & - & - & 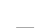 & - & - & - \\
\hline
\end{tabular}

(D)

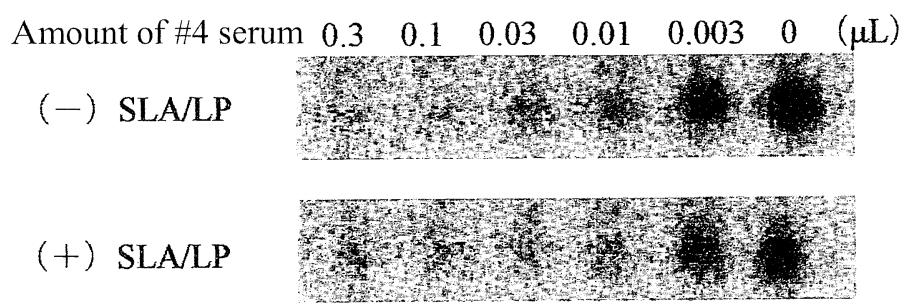

Fig. 3. TLC Pattern of $\left[{ }^{75} \mathrm{Se}\right] \mathrm{Sec}$ Produced with the SLA/LP Anti-peptide Globulin

(A), The four left lanes are with nonimmune globulin, and the four right lanes are with the immune globulin against SLA/LP peptide (rabbits). The amount of globulin used is shown below the lanes. (B), Influence of SLA/LP anti-peptide antibody on Sec synthesis. Enzyme preparations containing SecS were incubated with beads bound to anti-SLA/LP globulin (left) or to nonimmune globulin (right). Upper panel, $\left.{ }^{75} \mathrm{Se}\right] \mathrm{Sec}$ radioactive spot; lower panel, specific activity of Sec production. (C), The recombinant SLA/LP does not posses SecS activity. Upper panel, ${ }^{75}$ Se]Sec radioactive spot on TLC plate; lower panel, composition of the SLA/LP protein (30-100 $\mu \mathrm{g}$ ), bovine SecS, RNase inhibitor, and/or AIH sera (\#3 and \#4) or control serum (\#7) in the reaction mixture. (D), Influence of SLA/LP protein on the inhibition by AIH serum (\#4). Upper columns, no SLA/LP; lower columns, SLA/LP $0.6 \mu \mathrm{g}$. The amount of AIH serum (\#4) is displayed in the upper panel. The right side is without serum as positive control.

SLA/LP in human HEK293 cells. This was performed with immunoflorescence microscopy. First, we verified using SDS-PAGE that SLA/LP is expressed in HEK293 cell extracts. Figure 4A and 4B shows Western blots revealed with the SLA/LP antipeptide and anti-FLAG antibodies, respectively. Positive signals were obtained in extracts from the cells transfected with the expression vector (lane 1) but not in mock-transfected cells (lane 2) in Fig. 4A and 4B. An identical molecular mass of $57 \mathrm{kDa}$ was found in lane 1, which is higher than that predicted for SLA/LP because of the presence of the FLAG and 6 His tags. Figure $4 \mathrm{C}$ shows the presence of SLA/LP in the cytosol (lane 2), but not in the nucleus of HEK293-transfected cells (lane 1), whereas it could not be detected in the nucleus and the cytosol of mock-transfected cells (lanes 3 and 4). The presence of SLA/LP in the cytosol was confirmed by immunofluorescence microscopy (Fig. 5).

Thus we succeeded in the high expression of SLA/LP in the cytosol of HEK293 cells. Assuming that SLA/LP carries the SecS function, and given the high expression yield of this protein in the cytosol of HEK293 cells, we should expect to find a high selenocysteine level in the cell extract. However, as shown in Fig. 6, we could only detect selenocysteine amounts close to those in untransfected cells. This result did not support the hypothesis that SLA/LP is SecS.

Next, taking advantage of the flagged and 6His- 
tagged SLA/LP, we purified a protein complex containing SLA/LP with affinity chromatography from the cytosol of HEK293 cells expressing SLA/LP. Its components were analyzed. The silver staining of the gel is shown in Fig. 7. SLA/LP forms a complex with 70-kDa and 75-kDa proteins. Trypsin digests of the proteins in the complex were analyzed using LC-MS/MS, and the results show that the main complex contains HSP70 with SLA/LP.

A

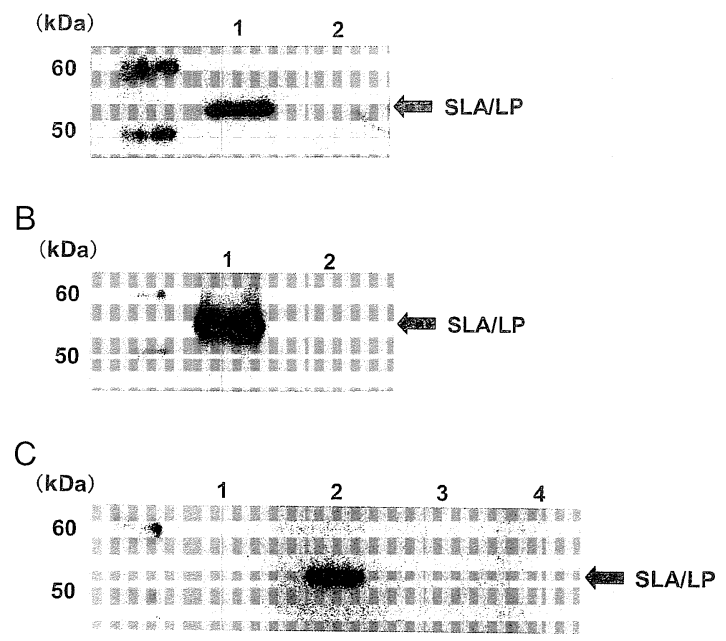

Fig. 4. SLA/LP Expression in HEK293 Cells Confirmed by Western Blotting

A, FLAG-SLA/LP reacted with SLA/LP anti-peptide antibody. Lane 1, lysate from cells transfected with the expression vector; lane 2, mock-transfected cells. B, FLAG-SLA/LP reacted with anti-FLAG peptide. Lane 1, lysate from cells transfected with the expression vector; lane 2, control cells. C, Distribution of SLA/LP in the cytosol of HEK293 cells. Lanes 1 and 2, cells with SLA/LP vector; lanes 3 and 4 , cells without vectors. Odd lanes contained nuclear fractions, even lanes the cytosol. The SLA/LP anti-peptide was used.
Table 1 shows the presence of HSP70 and/or tubulin in a complex with SLA/LP. Thus SLA/LP is present in the form of complexes with HSP70 and tubulin in the cytosol. However, we could not identify a function for the SLA/LP-HSP70 complex. Additionally, none of the proteins involved in the Sec translation machinery, such as SerRS, eEFsec or SBP2, was found associated in the complex with SLA/LP.

Figure 8 shows the pattern of Western blotting to detect antigens reacting with SLA/LP antipeptide antibody or AIH serum \#4. The antigen electrophoresed on the gel was a highly purified bovine $\mathrm{SecS}$. In the center two lanes, the primary antibody is the rabbit SLA/LP anti-peptide antibody. The primary antibody used in the far-right lane is AIH \#4 patient serum with the strongest inhibitory activity to SecS. The center lanes display a main band corresponding to a $35-\mathrm{kDa}$ protein and minor bands corresponding to 41,46 , and $58 \mathrm{kDa}$. The molecular mass of human SLA/LP is reported to be $47 \mathrm{kDa}$, but the $35-\mathrm{kDa}$ protein did not coin-

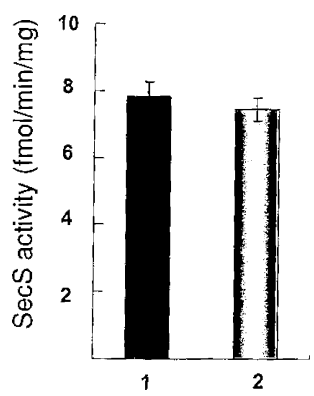

Fig. 6. SecS Activity in the Cytosol Containing High SLA/LP Levels

Left, cytosol expressing high SLA/LP levels; right, mocktransfected cells.

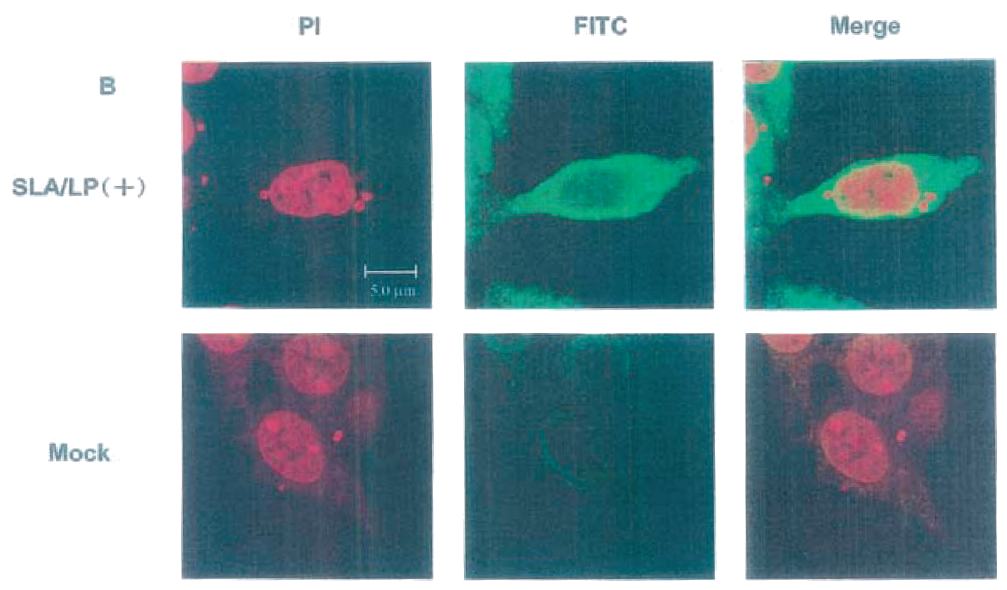

Fig. 5. Distribution of SLA/LP Shown with the Immunofluorescence Staining Method

The upper columns are from HEK293 cells expressing SLA/LP and the lower column shows mock-transfected cells. Left, nucleus stained with propidium iodide; center, with anti-FLAG antibody; right, merged. 
Table 1. Summary of Molecular Analyses of the SLA/LP Complex Using LC-MS/MS

\begin{tabular}{lclc}
\hline \hline gi(ID) & Protein mass & Protein & No. of peptides \\
\hline gi8393713 & 47449.16 & SLA/LP antigen isoform 1 & 3 \\
gi24432104 & 49432.24 & SLA/LP antigen isoform 2 & 20 \\
gi5123454 & 70280.13 & Heat shock 70-kDa protein 1A & 13 \\
gi4885431 & 70267.13 & Heat shock 70-kDa protein 1B & 1 \\
gi27436929 & 70760.46 & Heat shock 70-kDa protein 1-like & 8 \\
gi34419635 & 714404 & Heat shock 70-kDa protein 6 (HSP70B') & 4 \\
gi5729877 & 71082.31 & Heat shock 70-kDa protein 8 isoform 1 & 17 \\
gi24234686 & 53598.45 & Heat shock 70-kDa protein 8 isoform 2 & 2 \\
gi3029937 & 98621.73 & Heat shock 70-kDa protein 1, alpha isoform 1 & 1 \\
gi57013276 & 50803.86 & Tubulin, alpha, ubiquitous & 10 \\
gi29788785 & 50095.14 & Tubulin, beta polypepitde & 3 \\
gi29788766 & 50377.11 & Tubulin, beta polypeptide paralog & 4 \\
gi5174735 & 50255.17 & Tubulin, beta, 2 & 2 \\
gi50592996 & 50856.43 & Tubulin, beta, 4 & 5 \\
gi38455427 & 58400.95 & Chaperonin containing TCP1, subunit 4 (delta) & 1 \\
gi24307938 & 60088.98 & Chaperonin containing TCP1, subunit 5 (epsilon) & 1 \\
gi5453607 & 59842.12 & Chaperonin containing TCP1, subunit 7 isoform a & 1 \\
\hline
\end{tabular}

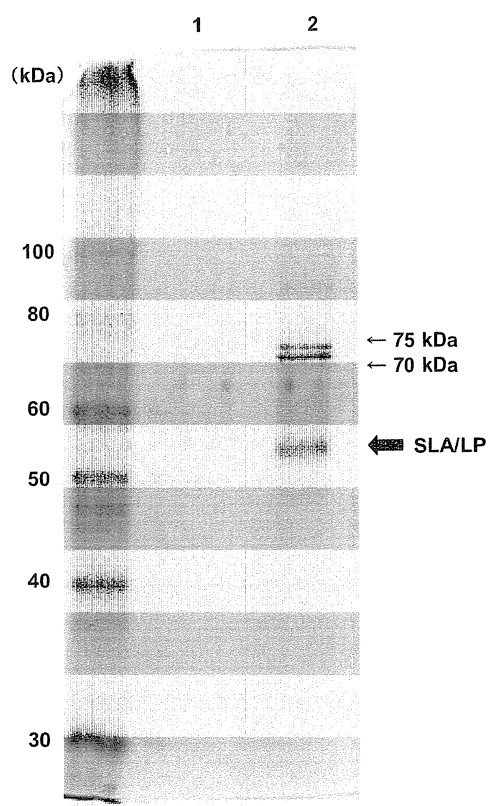

Fig. 7. Silver-stained Gel Showing the Components That Migrated as a Complex with SLA/LP

The SLA/LP complex was purified by affinity chromatography on anti-FLAG and Ni beads. Lane 1, mock extract; lane 2, extract from cells with high SLA/LP level. Protein markers are shown on the left.

cide with SLA/LP. However, it is possible that the $35-\mathrm{kDa}$ protein reacting with anti-SLA/LP may be a bovine SLA/LP or a derivative of bovine SLA/LP. In the far-right lane, the main band is a 54-kDa protein, with the other visible proteins being $35,41,50$, and $80 \mathrm{kDa}$. The $35-$ and $41-\mathrm{kDa}$ proteins are shared by both patterns. Meanwhile, the $35-\mathrm{kDa}$ protein band stained with anti-SLA/LP is also visible in the

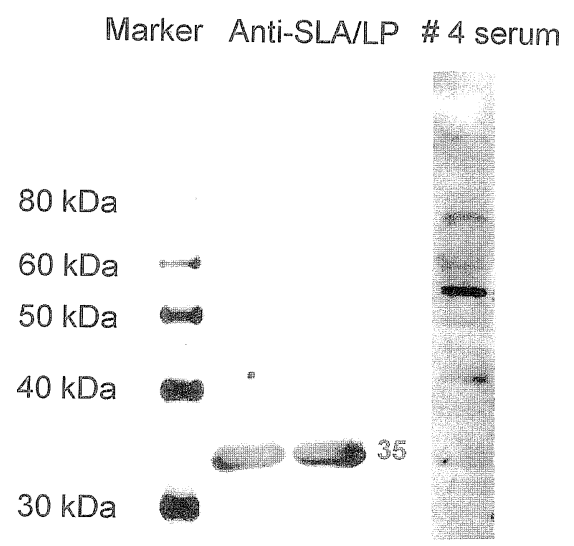

Fig. 8. Western Blotting

The highly purified bovine SecS fraction was electrophoresed on SDS-PAGE and blotted onto a PVDF membrane. Lane 1, protein markers; lanes 2 and 3, reaction with the SLA/LP anti-peptide antibody; lane 4 , reaction with a serum from an AIH patient \#4.

pattern stained with \#4 AIH serum, as well as other clear bands of 41, 50, 54, and $80 \mathrm{kDa}$. Anti-SLA/LP did not completely inhibit Sec production but serum \#4 did. Thus it is possible that the major $54-\mathrm{kDa}$ protein detected in the pattern stained with serum \#4 is SecS but that SLA/LP is not SecS.

\section{DISCUSSION}

In this study, we used two sera, \#4 AIH serum and anti-SLA/LP prepared in rabbits. The \#4 AIH serum, which may contain anti-SLA/LP, completely 
inhibited Sec production and we imagined that SLA/LP is SecS; however, SLA/LP anti-peptide antibody preparations resulted in only partial but not complete inhibition. SLA/LP itself did not show the activity of Sec formation and did not absorb inhibitory activity in \#4 AIH serum. From these results, we considered that SLA/LP itself is not SecS but a factor to enhance $\mathrm{Sec}$ production. The $C$ terminal of SLA/LP has antigenic activity. ${ }^{27)} \mathrm{We}$ prepared anti-SLA/LP against the $C$-terminal portion (position 402-420) of SLA/LP for use in this study. This $C$-terminal might not be the active site of $\mathrm{SLA} / \mathrm{LP}^{28)}$ but an antigenic site; therefore it is impossible to achieve direct inhibition of Sec formation by anti-peptide antibody. However, this antibody reacts and binds with SLA/LP. The enzyme preparation, from which SLA/LP was removed by absorption with a column binding SLA/LP antipeptide, showed SecS activity, as shown in Fig. 3B. This indicates that active SecS in the enzyme preparation is not SLA/LP. Meanwhile, we first reported that \#4 AIH serum completely inhibited Sec formation in vitro. AIH sera contain many types of antibody and we could not conclude whether antiSLA/LP in \#4 AIH serum inhibited Sec formation. It is possible that antibodies other than anti-SLA/LP in \#4 AIH serum inhibit Sec production, because \#4 AIH serum may contain anti-SerRS, anti-SeP synthetase, and anti-eEFsec, as well as anti-SecS.

To clarify the function of SLA/LP, which is a standard marker antigen of AIH type 1, we studied the effect of SLA/LP or anti-SLA/LP antibody on Sec production and also studied the distribution of SLA/LP in cellular organelles. We showed the inhibition of Sec synthesis by sera from AIH patients. We also showed the influence on Sec production of a serum directed against a $C$-terminal peptide of SLA/LP. Study of the distribution of SLA/LP in HEK293 cells established that it localizes in the cytosol. We could not show complete inhibition of Sec synthesis by the anti-SLA/LP peptide. We also could not attribute SecS activity to the native SLA/LP. Incomplete inhibition may depend on an unspecific interaction with globulin or impurities in the serum. We could not detect higher SecS activity in the cytosol of HEK293 cells transfected with the SLA/LP expression vector. These results show that SLA/LP is not the SecS, but there might be a deficiency in other components, such as tRNA ${ }^{\mathrm{Sec}}$, SerRS, and SPS.

We showed that SLA/LP is found in a complex with HSP70 or tubulin in HEK293 cells. Generally,
HSP70 play a role in protein transfer between cellular organelles and has refolding property of protein conformation. ${ }^{29)}$ Thus it is possible that SLA/LP has some other roles in the cytosol in association with HSP70 under normal physiologic conditions, different from its function in the Sec translation machinery under AIH disease conditions. Recently, the higher-order structure of HSP70 has been proposed to posses structural motifs sharing similarity to SerRS. ${ }^{30)}$

AIH is subdivided into two major types: AIH type 1 and AIH type 2. ${ }^{1)}$ AIH type 1 is characterized by the detection of SMA, ANA, ANCA, antiASGP-R, and anti-SLA/LP. Anti-SLA/LP is useful for the identification of individuals who are seronegative for ANA/SMA. AIH type 2 is characterized by the presence of autoantibodies against liver and kidney microsomal antigens (anti-LKM) and/or autoantibodies against liver cytosol 1 antigen; however, this subdivision is not completely clear, because mixed patterns are sometimes encountered. Patients themselves have individual autoantibody patterns according to the symptoms of AIH. AIH is a disease characterized by hypergammaglobulinemia involving many types of autoantibodies; therefore the patterns are dispersed and show many types of autoantibodies. We showed that sera \#3 and \#4 contain anti-SLA/LP, but also many other autoantibodies, although \#3 has the highest anti-SLA/LP among the four AIH patients. However, this high level of anti-SLA/LP does not mean that the levels of the other autoantibodies are also high (some autoantibody levels are high and others are low). We found the level of some autoantibodies higher in \#3 than in \#4. Thus SLA/LP is not the only autoantibody in AIH patients but one of many.

Generally, the number of components of the general translational machinery is higher in eukaryotes than in prokaryotes. This is also the case for the Sec production system and the peptide-elongation machinery. In mammals, the two functions of the $E$. coli specialized translation elongation factor SELB are divided into two molecules, SBP2 and $\left.\mathrm{EF}_{\mathrm{Sec}} ;{ }^{19}\right)$ the former recognizes the SECIS element on selenoprotein mRNAs, ${ }^{21)}$ while the latter is the elongation factor specific to Sec-tRNA ${ }^{\mathrm{Sec}}{ }^{20)}$ It is possible that other unknown proteins are involved to distinguish the UGA Sec and the UGA stop codons. In an earlier work, ${ }^{13)}$ we showed that the in vitro synthesis of $\mathrm{Sec}$ requires tRNA ${ }^{\mathrm{Sec}}$, SerRS, ATP, Ser, SPS, $\mathrm{H}_{2} \mathrm{Se}$, and $\mathrm{SecS}$. We also found that another protein may be necessary to produce a high amount of Sec in 
vitro (data not shown). Thus the Sec production system and the incorporation machinery are being clarified step by step, but there are still unknown participants in mammalian Sec synthesis. In this study, we showed that anti-SLA/LP incompletely inhibited Sec production and we could not establish that SLA/LP can inhibit the protection afforded to the Sec-tRNA ${ }^{\text {Sec }}$ by an elongation factor specific to Sec-tRNA ${ }^{\text {Sec }}{ }^{4)}$ Also the sequence of SLA/LP differs from SECp43, another protein found in a complex with the tRNA ${ }^{\mathrm{Sec}}{ }^{31)}$ In the future, we must establish whether the human SecS and SLA/LP share the same amino acid sequence. During the preparation of this manuscript, three reports attributing SecS function to SLA/LP with tRNA kinase were published. ${ }^{32-34)}$ However, our enzyme preparations, such as SerRS, SecS, and SPS, to produce Sec did not have any tRNA kinase activity or SLA/LP. We consider that our pathway to produce Sec should be the first pathway, composed of SecS and SPS, as in E. coli.

Acknowledgements This work was supported in part by a Grant (14572060 to TM) from the Ministry of Education, Sports, Culture, Science and Technology of Japan.

\section{REFERENCES}

1) Dalekos, G. N., Zachou, K., Liaskos, C. and Gatselis, N. (2002) Autoantibodies and defined target autoantigens in autoimmune hepatitis: an overview. Eur. J. Intern. Med., 13, 293-303.

2) Gelpi, C., Sontheimer, E. J. and Rodriguez-Sanchez, J. L. (1992) Autoantibodies against a serine tRNAprotein complex implicated cotranslational selenocysteine insertion. Proc. Natl. Acad. Sci. U.S.A., 89, 9739-9743.

3) Costa, M., Rodriguez-Sanchez, J. L., Czaja, A. J. and Gelpi, C. (2000) Isolation and characterization of cDNA encoding the antigenic protein of the human tRNP(Ser)Sec complex recognized by aitoantibodies from patients with type-1 autoimmune hepatitis. Clin. Exp. Immunol., 121, 364-374.

4) Yamada, K. (1995) A new translational elongation factor for selenocysteyl-tRNA in eukaryotes. FEBS Lett., 377, 313-317.

5) Wies, I., Brunner, S., Henninger, J., Heckel, J., Kanzler, S., Meer zum Buschenfelde, K. H. and Lohse, A. W. (2000) Identification of target antigen for SLA/LP autoantibodies in autoimmune hepatitis.
Lancet, 355, 1510-1515.

6) Ma, Y., Okamoto, M., Thomas, M. G., Bogdanos, D. P., Lopes, A. R., Portmann, B., Underhill, J., Durr, R., Mielu-Vergani, G. and Vergani, D. (2002) Antibodies to conformational epitopes of soluble liver antigen define a severe form of autoimmune liver disease. Hepatology, 35, 658-664.

7) Kernebeck, T., Lohse, A. W. and Grotzinger, J. (2001) A bioinformational function of the autoimmune hepatitis target antigen soluble liver antigen/liver pancreas. Hepatology, 34, 230-233.

8) Naparstek, Y. and Plotz, P. H. (1993) The role of autoantibodies in autoimmune disease. Ann. Rev. Immunol., 11, 79-104.

9) Keshan Disease Research Group (1979) Epidemiologic studies on the etiologic relationship of selenum. Chin. Med. J., 92, 477-482.

10) Allmang, C. and Krol, A. (2006) Selenoprotein synthesis, UGA does not end the story. Biochimie, 88, 1561-1571.

11) Mizutani, T., Goto, C. and Totsuka, T. (2000) Mammalian selenocysteine tRNA, its enzymes and selenophosphate. J. Health Sci., 46, 399-404.

12) Osaka, T., Beika, A., Hattori, A., Kohno, Y., Kato, K. H. and Mizutani, T. (2003) The protozoa dinoflagellate Oxyrrhis marina contains selenoproteins and the relevant translation apparatus. Bichem. Biophys. Res. Commun., 300, 236-240.

13) Mizutani, T., Kurata, H., Yamada, K. and Totsuka, T. (1992) Some properties of murine selenocysteine synthase. Biochem. J., 284, 827-834.

14) Bock, A. (2000) Biosynthesis of selenoproteins an overview. Biofactors, 11, 77-78.

15) Sturchler-Pierrat, C., Hubert, H., Totsuka, T., Mizutani, T., Carbon, P. and Krol, A. (1995) Selenocysteylation in eukaryotes necessitates the uniquely long aminiacyl acceptor stem of selenocysteine tRNA $^{\text {Sec }}$. J. Biol. Chem., 270, 18570-18574.

16) Amberg, R., Mizutani, T., Wu, X. Q. and Gross, H. J. (1996) Selenocysteine synthesis in mammalia: An identity switch from tRNA ${ }^{\text {Ser }}$ to tRNA ${ }^{\mathrm{Sec}}$. J. Mol. Biol., 263, 8-19.

17) Mizutani, T. and Goto, C. (2000) Eukaryotic selenocysteine tRNA has the $9 / 4$ secondary structure. FEBS Lett., 466, 173-182.

18) Mizutani, T., Kanaya, K. and Tanabe, K. (1999) Selenophosphate as a substrate for mammalian selenocysteine synthase, its stability and toxicity. Biofactors, 9, 27-36.

19) Atkins, J. and Gesteland, R. (2000) The $21^{\text {st }}$ amino acid selenocysteine. Nature, 407, 463-464.

20) Fagegaltier, D., Hubert, N., Yamada, K., Mizutani, T., Carbon, P. and Krol, A. (2000) Characterization 
of mSelB, a novel mammalian elongation factor for selenoprotein translation. EMBO J., 19, 4796-4805.

21) Zavacki, A. M., Mansell, J. B., Chung, M., Klimovitsky, B., Harney, J. W. and Berry, M. (2003) Coupled tRNA ${ }^{\mathrm{Sec}}$-dependent assembly of the selenocysteine decoding apparatus. Mol. Cell, 11, 773-781.

22) Herkel, J., Heidrich, B., Nieraad, N., Wies, I., Rother, M. and Lohse, A. W. (2002) Specifcity of autoantibodies to soluble liver antigen and liver/pancreas. Hepatology, 35, 403-408.

23) Tomita, M. and Tsong, T. Y. (1990) Selective production of hybridoma cells: antigenic-based preselection of B lymphocytes for electrofusion with lymphoma cells. Biochem. Biophys. Acta, 1055, 199206.

24) Usui, T., Kuno, T., Ueyama, H., Ohkubo, I. and Mizutani, T. (2006) Proximal HNF1 element is essential for the induction of human UDPglucuronosyltransferase $1 \mathrm{~A} 1$ by glucocorticoid receptor. Biochem Pharmacol., 71, 693-701.

25) Merril, C. R., Goldman, D., Sedman, S. A. and Ebert, M. H. (1981) Ultrasensitive stain for proteins in polyacrylamide gels shows regional variation in cerebrospinal fluid proteins, Science, 211, 14371438.

26) Natsume, T., Yamauchi, Y., Nakayama, H., Shinkawa, T., Yanagida, M., Takahashi, N. and Isobe, T. (2002) A direct nanoflow liquid chromatography-tandem mass spectrometry system for interaction proteomics. Anal. Chem., 74, 47254733.

27) Herkel, J., Heidrich, B., Nieraad, N., Wies, I.,
Rother, M. and Lohse, A. W. (2002) Specificity of antibodies to soluble liver antigen and liver/pancreas, Hepatology, 35, 403-408.

28) Kernebeck, T., Lohse, A. W. and Grotzinger, J. (2001) A biofunctional approach suggests the function of the autoimmune hepatitis target antigen soluble liver antigen/liver pancreas. Hepatology, 34, 230-233.

29) Feder, J. H., Ross, J. M., Solomon, J., Solomon, N. and Lindquist, S. (1992) The consequences of expressing hsp70 in Drosophila cells at normal temperatures. Genes Dev., 6, 1402-1413.

30) Carter, C. W. and Duax, W. L. (2002) Did tRNA synthetase classes arise on opposite strands of the same gene? Mol. Cell, 10, 705-708.

31) Ding, F. and Grabowski, P. J. (1999) Identification of a protein component of a mammalian tRNA ${ }^{\mathrm{Sec}}$ complex implicated in the decoding of UGA as selenocysteine. RNA, 5, 1561-1569.

32) Yuan, J., Palioura, S., Salazar, J. C., Su, D., O’Donoghue, P., Hohn, M. J., Cardoso, A. M., Whitman, W. B. and Söll, D. (2006) RNAdependent conversion of phosphoserine forms selenocysteine in eukaryotes and archaea. Proc. Natl. Acad. Sci. U.S.A., 103, 18923-18927.

33) Xu, X. M., Carlson, B. A., Mix, H., Zhang, Y., Saira, K., Glass, R. S., Berry, M. J., Gladyshev, V. N. and Hatfield, D. L. (2007) Biosynthesis of selenocysteine on its tRNA in eukaryotes. PLoS Biol., 5, e4, doi:10.1371/journal.pbio.0050004.

34) Ambrogelly, A., Palioura, S. and Söll, D. (2007) Natural expansion of the genetic code. Nat. Chem. Biol., 1, 29-35. 